\title{
Nonlinear Spring Model of Self-Organizing Map and its Chaotic Behavior
}

\author{
Haruna MATSUSHITA* and Yoshifumi NISHIO* \\ * Department of Electrical and Electronic Engineering, Tokushima University \\ 2-1 Minami-Josanjima, Tokushima 770-8506, Japan \\ Telephone: +81-88-656-7470, Fax: +81-88-656-7471, \\ Email: \{haruna, nishio\}@ee.tokushima-u.ac.jp
}

\begin{abstract}
The Self-Organizing Map (SOM) is an unsupervised neural network introduced by Kohonen and is a model simplifying self-organization process of the brain. However, SOM is still far away from the realization of the brain mechanism. In order to realize more powerful and more flexible mechanism, it is important to propose new models of the brain mechanism and to investigate their behaviors. In this study, as the first step to realize a new nonlinear spring model of SOM, we propose a simple one dimensional 2-neuron model connected by a nonlinear spring. We investigate its behavior under a simple assumption where input vectors are given to the model periodically. Computer simulated results show that the neurons oscillate chaotically.
\end{abstract}

\section{INTRODUCTION}

The Self-Organizing Map (SOM) is a subtype of artificial neural networks. It is trained using unsupervised learning to produce low dimensional representation of the training samples while preserving the topological properties of the input space. SOM is introduced by Kohonen in 1982 [1] and is a model simplifying self-organization process of the brain.

However, SOM is still far away from the realization of the brain mechanism. In order to realize more powerful and more flexible mechanism, it is important to propose new models of the brain mechanism and to investigate their behaviors.

In this study, as the first step to realize a new nonlinear spring model of SOM, we propose a simple one dimensional 2neuron model connected by a nonlinear spring. We investigate its behavior under a simple assumption where input vectors are given to the model periodically.

In Section II, we explain the learning algorithm of SOM. In Section III, we propose and explain the nonlinear spring model of SOM in detail. The behaviors of the proposed model are investigated by calculating Poincaré map and Lyapunov exponents in Section IV. Computer simulated results show that the neurons oscillate chaotically.

\section{Self-Organizing MAP (SOM)}

We explain the learning algorithm of the Self-Organizing Map (SOM). SOM consists of $m$ neurons located at a regular low-dimensional grid, usually a 2-D grid. The basic SOM algorithm is iterative. Each neuron $i$ has a $d$-dimensional weight vector $\boldsymbol{w}_{i}=\left(w_{i 1}, w_{i 2}, \cdots, w_{i d}\right)(i=1,2, \cdots, m)$. The initial values of all the weight vectors are given over the input space at random. The range of the elements of $d$-dimensional input data $\boldsymbol{x}_{j}=\left(x_{j 1}, x_{j 2}, \cdots, x_{j d}\right)(j=1,2, \cdots, N)$ are assumed to be from 0 to 1 .

(SOM1) An input vector $\boldsymbol{x}_{j}$ is inputted to all the neurons at the same time in parallel.

(SOM2) Distances between $\boldsymbol{x}_{j}$ and all the weight vectors are calculated. The winner, denoted by $c$, is the neuron with the weight vector closest to the input vector $\boldsymbol{x}_{j}$;

$$
c=\arg \min _{i}\left\{\left\|\boldsymbol{w}_{i}-\boldsymbol{x}_{j}\right\|\right\}
$$

where $\|\cdot\|$ is the distance measure, Euclidean distance.

(SOM3) The weight vectors of the neurons are updated as;

$$
\boldsymbol{w}_{i}(t+1)=\boldsymbol{w}_{i}(t)+h_{c, i}(t)\left(\boldsymbol{x}_{j}-\boldsymbol{w}_{i}(t)\right),
$$

where $t$ is the learning step. $h_{c, i}(t)$ is called the neighborhood function and is described as a Gaussian function;

$$
h_{c, i}(t)=\alpha(t) \exp \left(-\frac{\left\|\boldsymbol{r}_{i}-\boldsymbol{r}_{c}\right\|^{2}}{2 \sigma^{2}(t)}\right),
$$

where $\left\|\boldsymbol{r}_{i}-\boldsymbol{r}_{c}\right\|$ is the distance between map nodes $c$ and $i$ on the map grid, $\alpha(t)$ is the learning rate, and $\sigma(t)$ corresponds to the width of the neighborhood function. Both $\alpha(t)$ and $\sigma(t)$ decrease with time as follows;

$$
\alpha(t)=\alpha(0)\left(\frac{\alpha(T)}{\alpha(0)}\right)^{t / T}, \quad \sigma(t)=\sigma(0)\left(\frac{\sigma(T)}{\sigma(0)}\right)^{t / T},
$$

where $T$ is the maximum number of the learning.

(SOM4) The steps from (SOM1) to (SOM3) are repeated for all the input data.

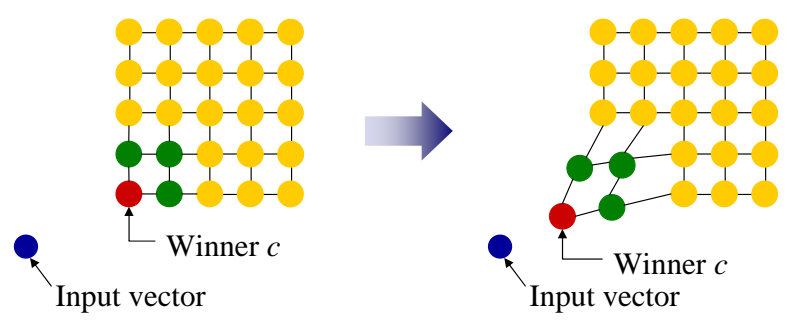

Fig. 1. Learning process of Self-Organizing Map. 


\section{Nonlinear Spring Model of SOM}

We consider a simple one dimensional 2-neuron model as the first step to realize the nonlinear spring model of SOM. The model is shown in Fig. 2. The two neurons are assumed to have the same mass $m$ and to be connected by a nonlinear spring with the natural length $l$ whose restoring force $F$ against the variation $x$ is represented by

$$
F=-b x^{3}
$$

where $b$ denotes the stiffness of the spring.

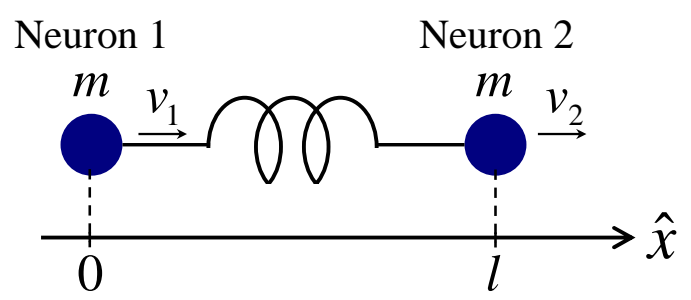

Fig. 2. Two-neuron nonlinear spring model of SOM.

Without loss of generality, we fix the position of the Neuron 1 as the origin of the $x$-coordinate. The position of the Neuron $2(\hat{x})$ and the velocities of the neurons $\left(v_{1}\right.$ and $\left.v_{2}\right)$ are chosen as the state variables. The motion equation of the model can be described as

$$
\left\{\begin{aligned}
\frac{d \hat{x}}{d t} & =v_{2} \\
m \frac{d v_{1}}{d t} & =-a v_{1}+b(\hat{x}-l)^{3} \\
m \frac{d v_{2}}{d t} & =-a v_{2}-b(\hat{x}-l)^{3}
\end{aligned}\right.
$$

where $a$ is the friction parameter. By changing the variables and parameters;

$$
\begin{aligned}
& \hat{x}-l=x, \quad v_{1}=\sqrt{\frac{b}{m}} y_{1}, \quad v_{2}=\sqrt{\frac{b}{m}} y_{2}, \\
& t=\sqrt{\frac{m}{b}} \tau, \quad k=\frac{a}{\sqrt{b m}},
\end{aligned}
$$

the normalized equations are given as

$$
\left\{\begin{aligned}
\frac{d x}{d \tau} & =y_{2} \\
\frac{d y_{1}}{d \tau} & =-k y_{1}+x^{3} \\
\frac{d y_{2}}{d \tau} & =-k y_{2}-x^{3}
\end{aligned}\right.
$$

Next, we model the learning process of the SOM by the external force by input vectors. When an input vector is given to the 2-neuron model as Fig. 3, the winner which is the neuron nearer to the input vector is attracted to the input with the following force;

$$
\hat{f}(t)=\hat{B} \sin \sqrt{\frac{b}{m}} t \quad\left(0 \leq t<\sqrt{\frac{m}{b}} \pi\right)
$$

where $t=0$ is the time when the input vector is given. The shape of this function is shown in Fig. 4. Note that the other neuron does not receive a direct effect from the input vector.

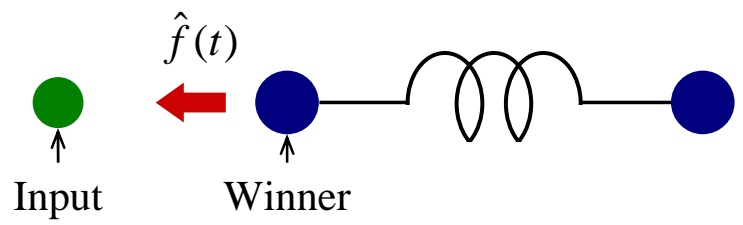

Fig. 3. Input vector and winner.

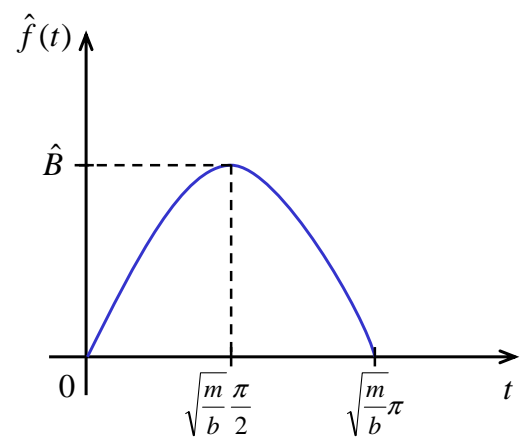

Fig. 4. Force by external input vector.

In this study, in order to investigate the simplest learning process of the 2-neuron model, we concentrate on the case that input vectors are given to the right-hand side and the lefthand side of the model alternately with the fixed frequency $\sqrt{b / m} /(2 \pi)$. In this case, the motion equation is modified as

$$
\left\{\begin{aligned}
\frac{d x}{d \tau} & =y_{2} \\
\frac{d y_{1}}{d \tau} & =-k y_{1}+x^{3}-f(\tau) \\
\frac{d y_{2}}{d \tau} & =-k y_{2}-x^{3}-f(\tau-\pi),
\end{aligned}\right.
$$

where

$$
f(\tau)=\frac{B}{2}(\sin \tau+|\sin \tau|)
$$

and

$$
B=\frac{\hat{B}}{b} .
$$

The shape of $f(\tau)$ is shown in Fig. 5 .

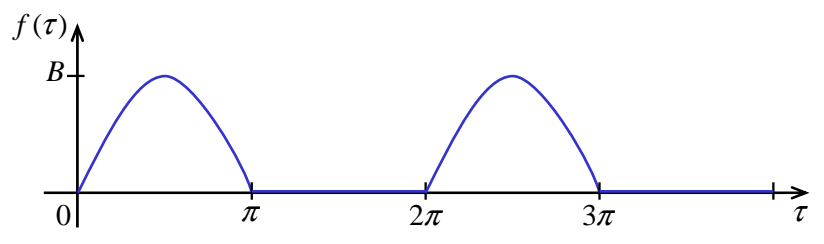

Fig. 5. Force by periodic external input vector. 


\section{Simulation Results}

In this section, we show some computer calculation results obtained by using Runge-Kutta method with time step $\delta t=$ $2 \pi / 100$ for Eq. 10.

Figure 6 shows time series of $x$, namely the position of Neuron 2 in Fig. 2. Figure 6(a) is obtained for the parameters $k=0.27$ and $B=8.5$, while Figure $6(\mathrm{~b})$ is for $k=0.1$ and $B=12$. Although we can see some periodicity in the waveform in Fig. 6(a), the movement is completely nonperiodic in Fig. 6(b). The attractors corresponding to the time series are shown in Fig. 7. We can confirm that the orbits of the solution look chaos.

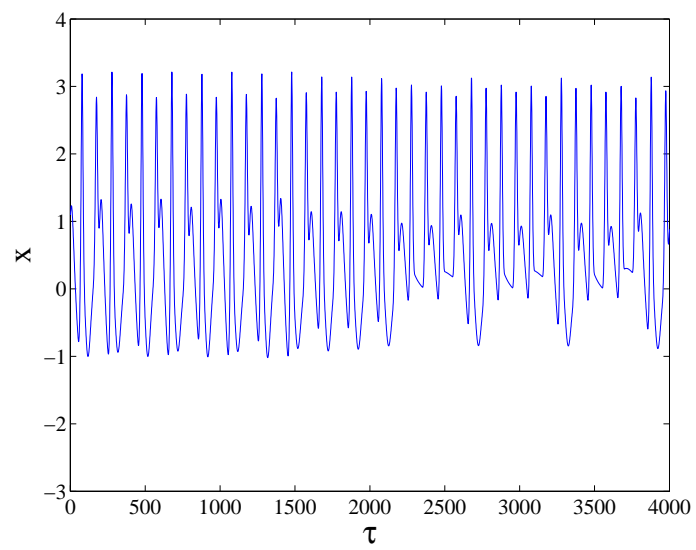

(a)

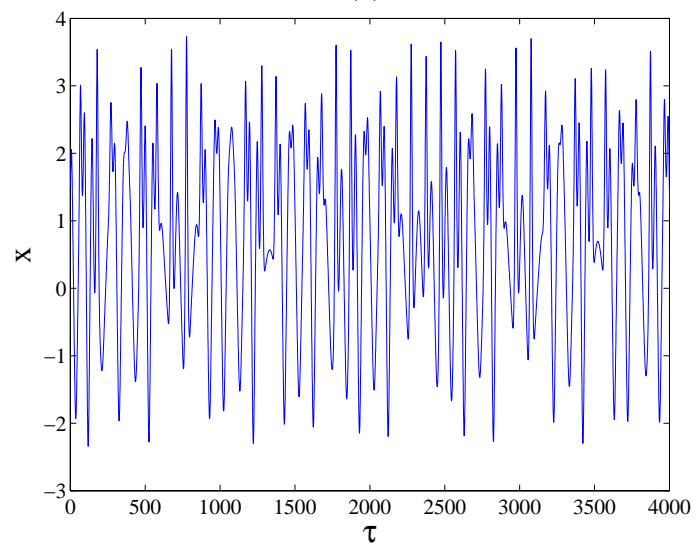

(b)

Fig. 6. Time series of nonlinear spring model. (a) $k=0.27, B=8.5$. (b) $k=0.1, B=12$.

In order to investigate the chaotic behavior of the model in detail, we define the Poincaré section as $\tau=2 n \pi$ and plot the discrete data on the Poincaré section onto $x-y_{2}$ plane. Figure 8 shows the Poincaré map corresponding to the attractors in Fig. 7. We can see that the Poincaré map is folded and has the shape like strange attractors [2].

In order to confirm the generation of chaos, we calculate the Lyapunov exponents of the attractors using the algorithm of Shimada-Nagashima [3]. The Jacobian matrix are obtained by calculating the variational equations of Eq. (10) together [4].

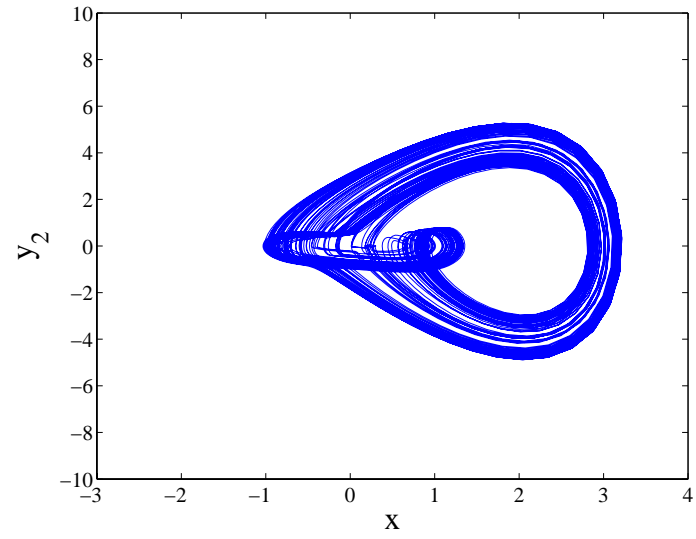

(a)

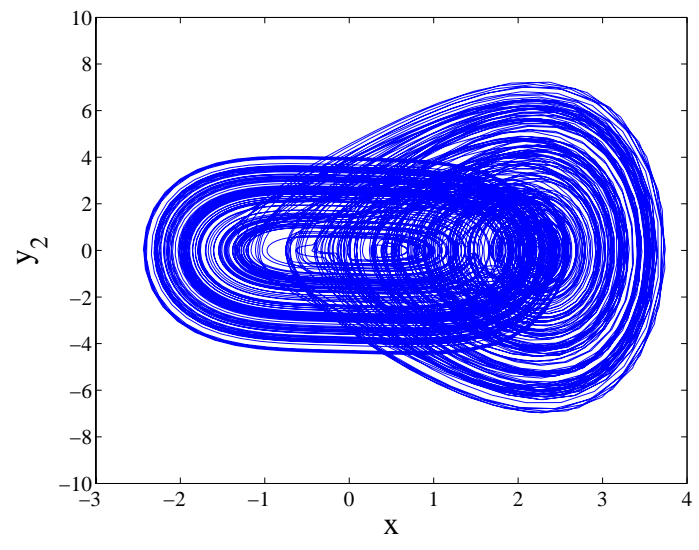

(b)

Fig. 7. Attractor of nonlinear spring model. (a) $k=0.27, B=8.5$. (b) $k=$ $0.1, B=12$.

Figure 9 shows the calculated largest Lyapunov exponents. Because the Lyapunov exponents are positive for both cases, we can say that the attractors are chaos.

\section{CONCLusions}

In this study, as the first step to realize a new nonlinear spring model of SOM, we have proposed a simple one dimensional 2-neuron model connected by a nonlinear spring. We have investigated its behavior under a simple assumption where input vectors are given to the model periodically. We have confirmed that the neurons oscillate chaotically.

\section{REFERENCES}

[1] T. Kohonen, Self-organizing Maps, Berlin, Springer, vol. 30, 1995.

[2] Y. Ueda, The Road to Chaos, Santa Cruz, Aerial Press, 1992.

[3] I. Shimada and T. Nagashima, "A Numerical Approach to Ergodic Problem of Dissipative Dynamical Systems," Progress of Theoretical Physics, vol. 61, No. 6, pp. 1605-1616, 1979.

[4] H. Kawakami, "Bifurcation of Periodic Responses in Forced Dynamic Nonlinear Circuits: Computation of Bifurcation Values of the System Parameters," IEEE Trans. Circuits and Systems, vol. CAS-31, No. 3, pp. 248-260, 1984. 


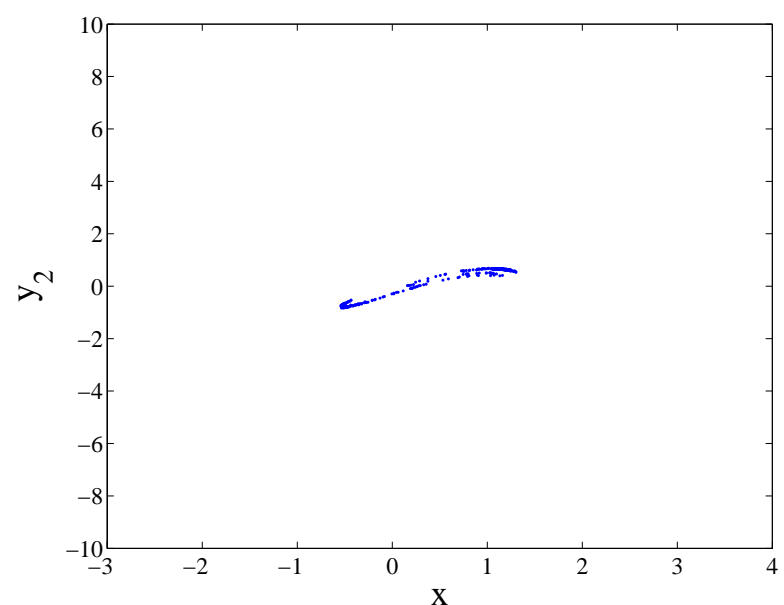

(a)

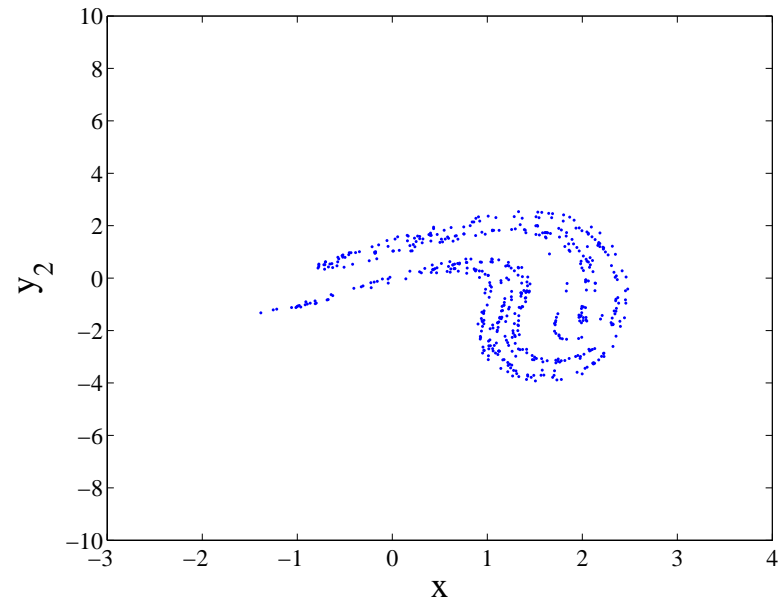

(b)

Fig. 8. Poincaré map of nonlinear spring model. (a) $k=0.27, B=8.5$. (b) $k=0.1, B=12$.

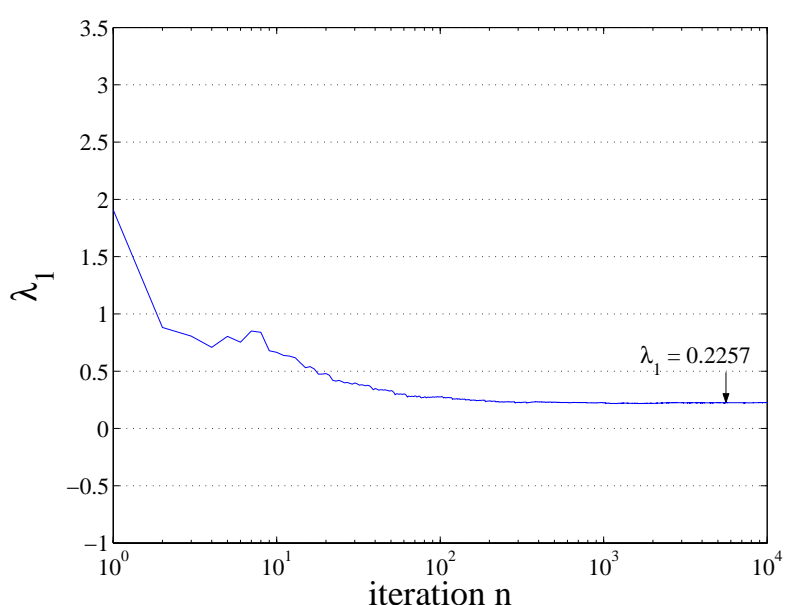

(a)

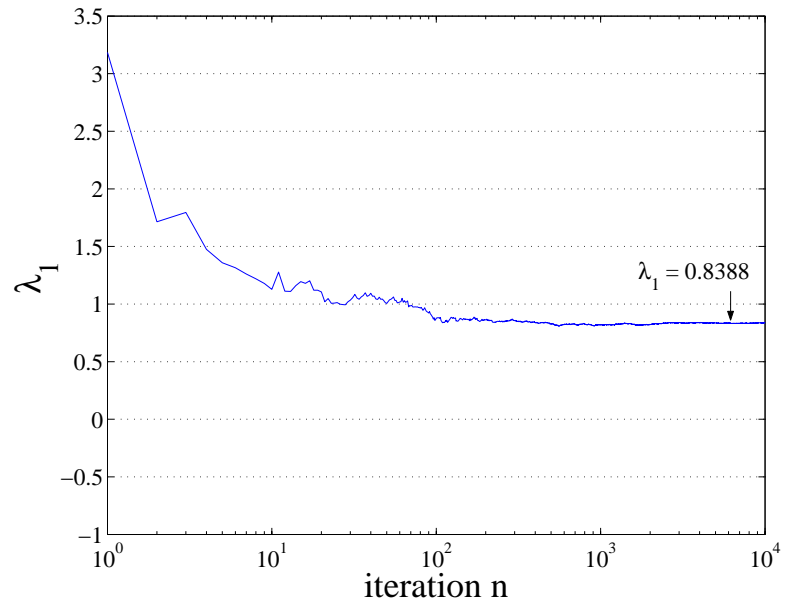

(b)

Fig. 9. Lyapunov exponent of attractor. (a) $k=0.27, B=8.5$. (b) $k=$ $0.1, B=12$. 